\title{
Larger aortic reconstruction corresponds to diminished left pulmonary artery size in patients with single-ventricle physiology
}

\author{
Lakshmi P. Dasi, PhD, ${ }^{\text {a }}$ Kartik S. Sundareswaran, $\mathrm{PhD},{ }^{\text {a }}$ Colleen Sherwin, BS, ${ }^{\text {a }}$ Diane de Zelicourt, MS, \\ Kirk Kanter, MD, ${ }^{\mathrm{b}}$ Mark A. Fogel, $\mathrm{MD},{ }^{\mathrm{c}}$ and Ajit P. Yoganathan, $\mathrm{PhD}^{\mathrm{a}}$
}

\begin{abstract}
Background: Pulmonary artery size is a crucial determinant of hemodynamic energy loss in total cavopulmonary connections. We investigated the effect of aortic arch reconstruction on left pulmonary artery size based on their anatomic proximity.
\end{abstract}

\begin{abstract}
Methods: Thirty-two patients undergoing the Fontan operation, 16 with hypoplastic left heart syndrome and 16 with non-hypoplastic left heart syndrome, were selected from the multicenter Fontan magnetic resonance imaging database at the Georgia Institute of Technology. The 16 datasets were consecutive with full anatomic reconstructions of the total cavopulmonary connection and aortic arch with no artifacts. The size of the aorta along the transverse arch and left pulmonary artery size in the region below the aortic arch was quantified by using a previously validated skeletonization technique.

Results: The transverse aortic and left pulmonary artery measurements (median, maximum, and minimum, respectively) for non-hypoplastic left heart syndrome were $2.2,3.1$, and $1.5 \mathrm{~cm} / \mathrm{m}$ and $1.2,1.6$, and $0.2 \mathrm{~cm} / \mathrm{m}$, respectively, compared with $2.5,4.1$, and $2.0 \mathrm{~cm} / \mathrm{m}$ and $0.9,1.5$, and $0.4 \mathrm{~cm} / \mathrm{m}$ for patients with hypoplastic left heart syndrome. Thus the transverse aortic diameter of patients with hypoplastic left heart syndrome was, on average, $24 \%$ greater than that for patients with non-hypoplastic left heart syndrome $(P<.05)$, whereas the left pulmonary artery diameter of patients with hypoplastic left heart syndrome was smaller than that of patients with non-hypoplastic left heart syndrome $(P<.05)$. Regression analysis showed a significant negative correlation $(P<.05)$ between aortic and left pulmonary artery diameters in both the hypoplastic left heart syndrome and non-hypoplastic left heart syndrome groups. However, when the study population was regrouped into reconstructed aorta and nonreconstructed aorta groups, the negative correlation was only significant for patients with reconstructed aortas, regardless of ventricular pathology $(P<.02)$.
\end{abstract}

Conclusions: Stage 1 aortic reconstruction procedures that result in a large aorta limit left pulmonary artery size in patients undergoing the Fontan operation. (J Thorac Cardiovasc Surg 2010;139:557-61)

Pulmonary artery stenosis is a common lesion that limits the efficacy of the Fontan operation, ${ }^{1-3}$ particularly because patients with single-ventricle Fontan physiology need an energy-efficient circulatory system for minimizing the work load on the single ventricle. ${ }^{4,5}$ Although there exist several studies that focus on characterizing the hemodynamics of various stages leading to Fontan physiology, ${ }^{4-7}$ there is yet no explanation of the cause of pulmonary artery stenosis.


Technology, Atlanta, Ga; Emory University, ${ }^{\mathrm{b}}$ Atlanta, Ga; and the Division of Cardiology, ${ }^{c}$ Children's Hospital of Philadelphia, Philadelphia, Pa.

Lakshmi P. Dasi's current affiliation is Department of Mechanical Engineering, Colorado State University, Fort Collins, Colo.

Disclosures: None.

We gratefully acknowledge the Bioengineering Research Partnership (BRP) grant from the National Institutes of Health (HL67622).

Received for publication Feb 21, 2009; revisions received June 24, 2009; accepted for publication Aug 10, 2009; available ahead of print Nov 2, 2009.

Address for reprints: Ajit P. Yoganathan, PhD, Wallace H. Coulter School of Biomedical Engineering, Georgia Institute of Technology and Emory University, Room 2119 U. A. Whitaker Building, 313 Ferst Dr, Atlanta, GA 30332-0535 (E-mail: ajit.yoganathan@bme.gatech.edu).

0022-5223/\$36.00

Copyright (c) 2010 Published by Elsevier Inc. on behalf of The American Association for Thoracic Surgery

doi:10.1016/j.jtcvs.2009.08.023
}

Our previous article ${ }^{8}$ and that of Senzaki and colleagues ${ }^{9}$ showed that a limiting pulmonary artery causes significant energy losses that affect the resting cardiac output or ventricular afterload.

With the large anatomic database of patients undergoing the Fontan operation available at the Georgia Institute of Technology, it is now possible to study how various vessels might interact with one another with respect to their complex anatomies. The present work focuses on a possible physical interaction between the left pulmonary artery (LPA) and aortic arch because the 2 structures are intimately close and connected; that is, the aortic arch passes over the LPA, as depicted in the full anatomic reconstruction shown in Figure 1. Noticing that the aorta shown in Figure 1 is large and has been reconstructed during the first stage of the 3 Fontan operations, we hypothesize that stenosis in the LPA might be caused by the physical constraints placed on the LPA by the reconstructed aortic geometry. The present work tests this hypothesis in 2 distinct classes of patients with single-ventricle physiology, those with hypoplastic left heart syndrome (HLHS) and those with non-hypoplastic left heart syndrome (NHLHS), because aortic reconstruction is performed in both of these groups. The results of this 


\begin{tabular}{|l} 
Abbreviations and Acronyms \\
BSA $=$ body surface area \\
HLHS $=$ hypoplastic left heart syndrome \\
LPA $=$ left pulmonary artery \\
MRI $=$ magnetic resonance imaging \\
NHLHS $=$ non-hypoplastic left heart syndrome \\
NRA $=$ nonreconstructed aorta \\
RA $=$ reconstructed aorta \\
TCPC $=$ total cavopulmonary connection
\end{tabular}

work demonstrate how the 3 stages of the Fontan operation and their outcomes are not entirely independent. Specifically, although the aortic reconstruction might be optimal at the time of the operation, it could diminish the efficacy of an operation at a later stage, calling for a more sophisticated planning of the entire course of palliation.

\section{MATERIALS AND METHODS}

Thirty-two patients, 16 each with HLHS and NHLHS, were selected from a magnetic resonance imaging (MRI) database of patients undergoing the Fon$\tan$ operation (http://fontan.bme.gatech.edu). The database is part of a National Institutes of Health-funded ongoing study for understanding Fontan hemodynamics. All patients were imaged either at Children's Hospital of Philadelphia or at Emory University/Children's Healthcare of Atlanta. Informed consent was obtained from all patients, and all study protocols complied with the institutional review boards of the participating hospitals and the Georgia Institute of Technology. The inclusion criteria for this study were (1) availability of axial MRI images to reconstruct the total cavopulmonary connection (TCPC) and aortic arch and (2) availability of clinical information necessary to categorize each study group. Anatomic reconstructions with visible artifacts (some geometries had loss of MRI signal because of the presence of "clips" in the vessels from surgical intervention) were excluded from the study group.

Clinical details of the studied patient population are provided in Table 1. Although the Fontan database has more than 200 MRI datasets, this study was limited to the first 16 HLHS and NHLHS datasets, which proved to be statistically sufficient. Although each patient has a unique diagnosis (Table 1), the HLHS/NHLHS status grouping differentiates them with respect to the underlying congenital defect, as done previously. ${ }^{8,10,11}$ For all 32 patients, the anatomies of the TCPC and aortic arch were reconstructed by using standard segmentation and reconstruction techniques. ${ }^{12,13}$ The effect of the aortic arch on the LPA was quantified by measuring the crosssectional areas of the 2 vessels at the closest approach in the arch. The diameter at the cross-section is defined as the diameter of a circular crosssection for that area. This was performed by using the skeletonization method depicted in Figure 2. The skeletonization method has been previously used to study the geometric characteristics of the TCPC. ${ }^{10}$

Because the data were nonnormally distributed and corresponded to a 2sample population (HLHS vs NHLHS or reconstructed aorta [RA] vs nonreconstructed aorta [NRA]), the nonparametric Mann-Whitney test was used to examine statistical significance among the various geometric parameters evaluated.

\section{RESULTS}

The results consist of statistical analysis of the aortic and LPA diameter measurements of the 32 patients normalized by the square root of the patient's body surface area (BSA) based on available allometric relationship. ${ }^{14}$ The



FIGURE 1. Example of a reconstructed aorta overlaying a narrowed left pulmonary artery.

data are analyzed between the HLHS versus NHLHS patient groups and also simultaneously between the RA versus NRA groups. We define the RA group as undergoing reconstruction that involves the aortic arch from the ascending portion to the ductus insertion region. For instance, a repair of an aortic coarctation is not defined as an RA. This is necessary to precisely determine whether an RA is a factor in LPA narrowing independent of underlying ventricular abnormality.

Figure 3 shows a scatter plot of the transverse aortic and LPA diameters normalized by BSA ${ }^{1 / 2}$ for all the patients. 
TABLE 1. Clinical diagnoses of the 32 patients selected for this study

\begin{tabular}{|c|c|c|c|c|}
\hline Patient no. & Diagnosis & Aorticreconstruction & $\operatorname{BSA}\left(\mathrm{m}^{2}\right)$ & Age (y) \\
\hline \multicolumn{5}{|c|}{ Patients with HLHS } \\
\hline 1 & DORV, MA, HLHS & Yes & 1.22 & 12 \\
\hline 2 & HLHS & Yes & 1.68 & 14 \\
\hline 3 & TGA, SLL, LAVV atresia, severe sub-PS with LPA occlusion & No & 1.30 & 19 \\
\hline 4 & HLHS & Yes & 0.94 & 8 \\
\hline 5 & HLHS, ASD, LSVC to CS, clotted RSVC & Yes & 1.43 & 17 \\
\hline 6 & D-transposition, LV hypoplasia, left SVC to coronary sinus & Yes & 1.9 & 16 \\
\hline 7 & HLHS, ASD & Yes & 1.23 & 12 \\
\hline 8 & HLHS & Yes & 1.358 & 16 \\
\hline 9 & HLHS & Yes & 0.994 & 10 \\
\hline 10 & DORV, subaortic VSD, PS, hypoplastic MV and LV & Yes & 2.05 & 19 \\
\hline 11 & LTGA, SLL, DORV, VSD, PS & Yes & 0.69 & 9 \\
\hline 12 & HLHS & Yes & 0.83 & 6 \\
\hline 13 & HLHS & Yes & 0.963 & 9 \\
\hline 14 & HLHS & Yes & 0.81 & 5 \\
\hline 15 & HLHS, bilateral SVC & Yes & 0.91 & 7 \\
\hline 16 & HLHS, ASD & Yes & 0.83 & 6 \\
\hline \multicolumn{5}{|c|}{ Patients with NHLHS } \\
\hline 17 & HRHS, Ebstein's anomaly of tricuspid & No & 1.02 & 8 \\
\hline 18 & TA, VSD & No & 1.32 & 10 \\
\hline 19 & SV with subaortic and aortic valve stenosis & Yes & 1.045 & 10 \\
\hline 20 & TA, pulmonary stenosis, transposition & No & 1.9 & 24 \\
\hline 21 & HRV, TA, TGA, VSD & Yes & 0.88 & 8 \\
\hline 22 & TA, VSD, bilateral SVC & No & 1.84 & 20 \\
\hline 23 & HRV, TA & No & 0.69 & 5 \\
\hline 24 & HRV, TGA, TA, VSD, LPA hypoplasia & No & 0.58 & 2 \\
\hline 25 & TA, VSD & No & 0.872 & 7 \\
\hline 26 & PA, IVS & No & 1.177 & 11 \\
\hline 27 & SV DI-LV, VPS-TGA & Yes & 1.064 & 9 \\
\hline 28 & SV-PA, IVS, HRV & No & 1.04 & 12 \\
\hline 29 & Subpulmonary stenosis, HRV, TGA, VSD & Yes & 0.813 & 6 \\
\hline 30 & TA, D-transposition, hypoplastic arch, small VSD & Yes & 0.74 & 7 \\
\hline 31 & Pulmonary atresia, HRV & No & 1.49 & 15 \\
\hline 32 & Single ventricle-DORV, VSD, aortic arch hypoplasia & Yes & 1.41 & 14 \\
\hline
\end{tabular}

$B S A$, Body surface area; $H L H S$, hypoplastic left heart syndrome; $D O R V$, double-outlet right ventricle; $M A$, mitral atresia; $T G A$, transposition of the great arteries; $S L L$, situs solitus; $L A V V$, left atrioventricular valve; $P S$, pulmonary stenosis; $L P A$, left pulmonary artery; $A S D$, atrial septal defect; $L S V C$, left superior vena cava; $C S$, coronary sinus; $R S V C$, right superior vena cava; $L V$, left ventricle; $S V C$, superior vena cava; $N H L H S$, non-hypoplastic left heart syndrome $V S D$, ventricular septal defect; $M V$, mitral valve; $L T G A$, levo transposition of the great arteries; $H R H S$, hypoplastic right heart syndrome; $T A$, tricuspid atresia; $S V$, single ventricle; $H R V$, hypoplastic right ventricle; $P A$, pulmonary atresia; $I V S$, intact ventricular septum; $D I$, double inlet; $V P S$, valvar pulmonary stenosis.

The figure also compares these data against average values for healthy children (approximately $1.0 \mathrm{~cm} / \mathrm{m}$ for the LPA and $1.5 \mathrm{~cm} / \mathrm{m}$ for the transverse aorta). ${ }^{14}$ For the NHLHS patient group the median, maximum, and minimum values for the aortic and LPA measurements were 2.2, 3.1 , and $1.5 \mathrm{~cm} / \mathrm{m}$ and $1.2,1.6$, and $0.2 \mathrm{~cm} / \mathrm{m}$, respectively. For the HLHS patient group, these scatter characteristics were $2.5,4.1$, and $2.0 \mathrm{~cm} / \mathrm{m}$ and $0.9,1.5$, and $0.4 \mathrm{~cm} / \mathrm{m}$ for the aortic and LPA measurements, respectively.

The mean of the normalized aortic and LPA diameters are shown in Figure 4 and are compared statistically. The aortic diameter for the HLHS patient group was statistically greater $(P<.05)$ than that for the NHLHS patient group by about $24 \%$. Figure 4 also shows that the LPA diameter for the HLHS patient group is smaller than that for the NHLHS patient group $(P<.05)$. Figure $4, B$, shows the same compar- ison between aortic and LPA diameters between patients for the RA versus NRA groups. Aortic diameter is statistically greater and LPA diameter is statistically smaller in patients with an RA versus those with an NRA.

Figure 5 depicts results from regression analysis of the data between the HLHS versus NHLHS and RA versus NRA groups, respectively, where the LPA diameter is plotted as a function of the aortic diameter. Figure $5, A$, shows that in both the HLHS and NHLHS groups there is a statistically significant $(P<.05)$ association between increasing aortic diameter causing decreasing LPA diameter. Figure $5, B$, however shows that the NRA group does not have any statistically significant association between increasing aortic diameter and decreasing LPA diameter. In addition, the $P$ value for statistical significance for the RA group is the smallest $(P=.012)$. 


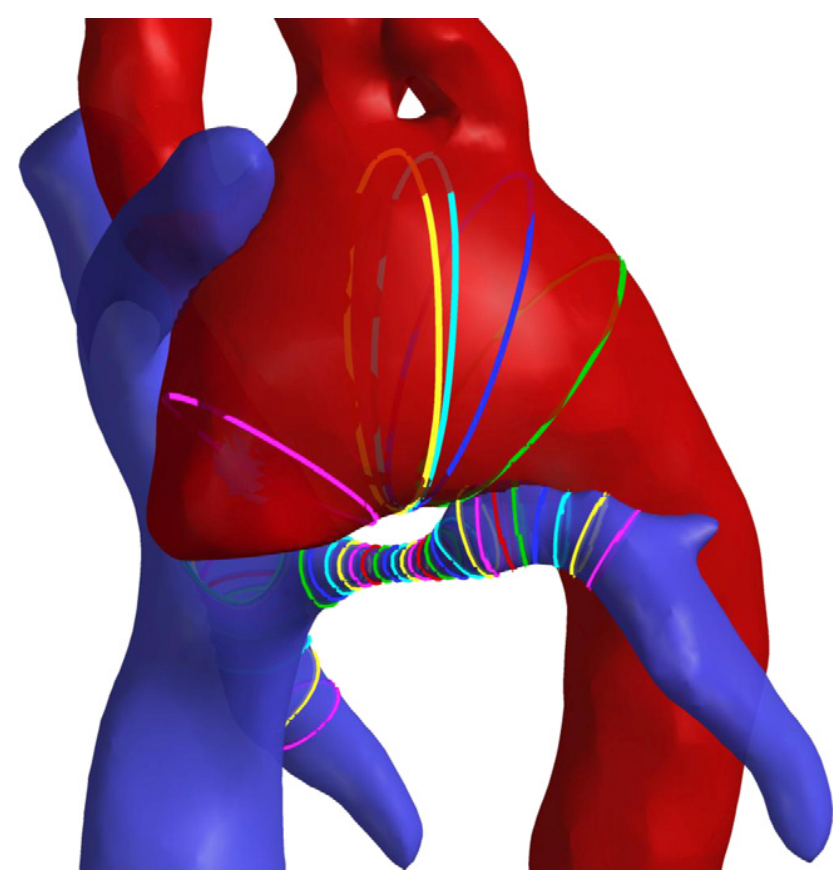

FIGURE 2. Skeletonization view of the aorta-left pulmonary artery overlap region. Ribbons are cross-sections of the respective vessels. The minimum left pulmonary artery area was located, followed by the area of the transverse aortic cross-section closest to the minimum left pulmonary artery location.

\section{DISCUSSION}

Figures 1 and 2 clearly depict the possible effect a large aorta can have on the LPA that is anatomically situated right below the arch. Note that all of these reconstructions are blood volumes only; that is, the surfaces in Figures 1 and 2 are the inner walls of the lumen. Therefore the small gap seen between the aorta and the LPA in Figure 2 is in fact occupied by the vessel wall thickness. Examination of the raw MRI images confirmed this. From Figure 3, notice that almost all the patients have aortas bigger than that seen in a healthy child, ${ }^{14}$ whereas the LPA is distributed equally about the normal range

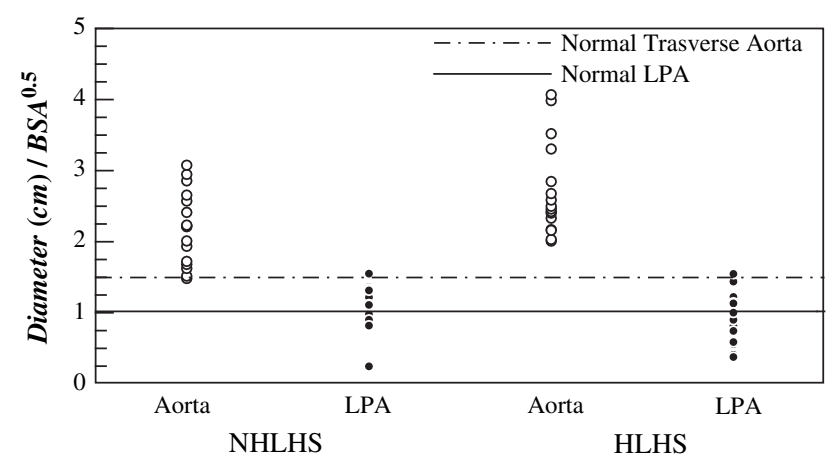

FIGURE 3. Scatter plot of measured transverse aortic and left pulmonary artery (LPA) diameters for the non-hypoplastic left heart syndrome (NHLHS) and hypoplastic left heart syndrome (HLHS) patient groups. The solid and dashed lines represent normal LPA and transverse aortic dimensions, respectively, in healthy children. BSA, Body surface area.
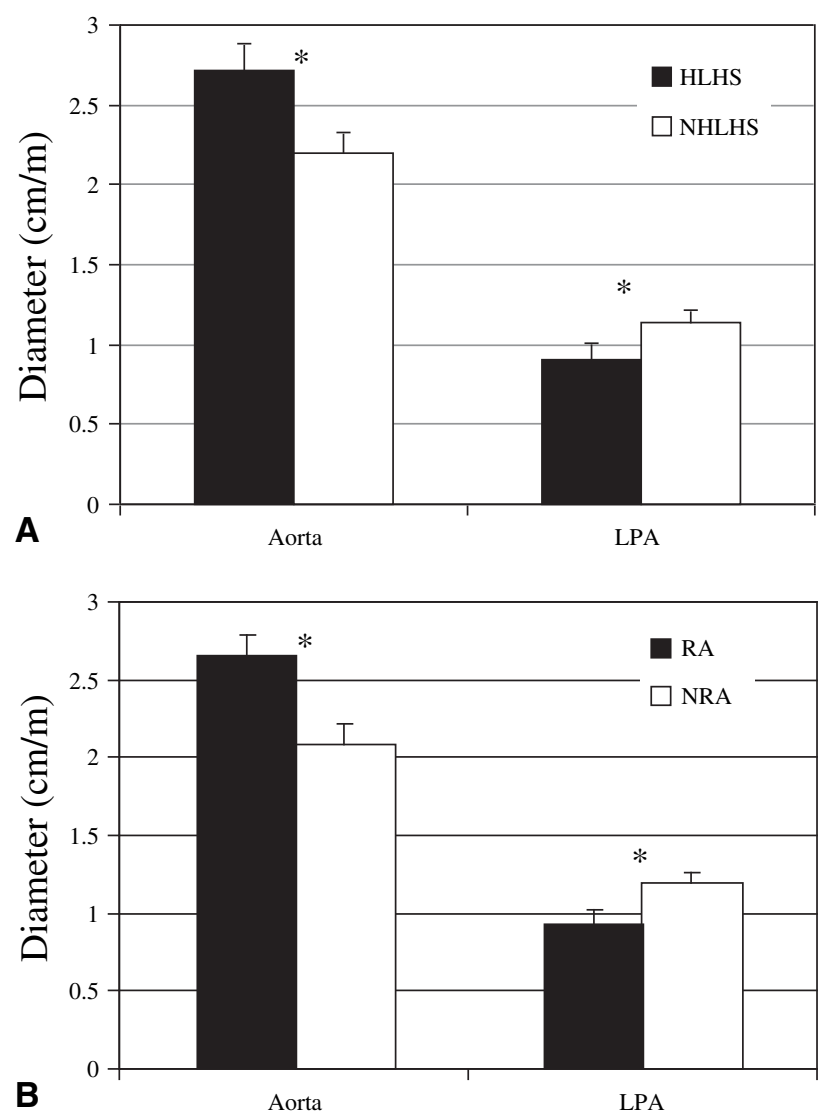

FIGURE 4. Comparison between aortic and left pulmonary artery (LPA) sizes at the junction across the hypoplastic left heart syndrome (HLHS) and non-hypoplastic left heart syndrome (NHLHS) patient groups (A) and patients with reconstructed arteries $(R A)$ and nonreconstructed arteries (NRA) (B), respectively. $* P<.05$.

for patients with HLHS and skewed in patients with NHLHS. To give a feel for the range of aortic sizes, the smallest aorta in the entire dataset was the most normal (ie, healthy), with a size of $1.5 \mathrm{~cm} / \mathrm{m}$ (the same as for healthy patients ${ }^{14}$ ), whereas the largest aorta was 2.7 times bigger at $4.1 \mathrm{~cm} / \mathrm{m}$. This implies that this enlarged aorta provides 7.5 times more flow area. The maximum aortic size was $3.7 \mathrm{~cm}$ (patient BSA was $0.83 \mathrm{~m}^{2}$ ), and the patient would have $225 \%$ more flow area when an adult (based on an adult aorta of $2.5 \mathrm{~cm}$ ). An enlarged aorta of such proportions itself creates significant energy losses because of the expansion and contraction of the aortic flow, thus further increasing the energetic load on the single ventricle. The smallest LPA was $0.2 \mathrm{~cm} / \mathrm{m}$, which provides roughly 25 times lower flow area than in a healthy LPA. The scatter of LPA in the NHLHS group shows that there was 1 patient with an abnormally low LPA value, whereas the rest were skewed above the normal healthy value. For the HLHS group, the LPA scatter was about equally distributed about the healthy/normal value.

Statistical analysis represented in Figure 4 clearly shows that the aortic diameter was larger for the HLHS group 


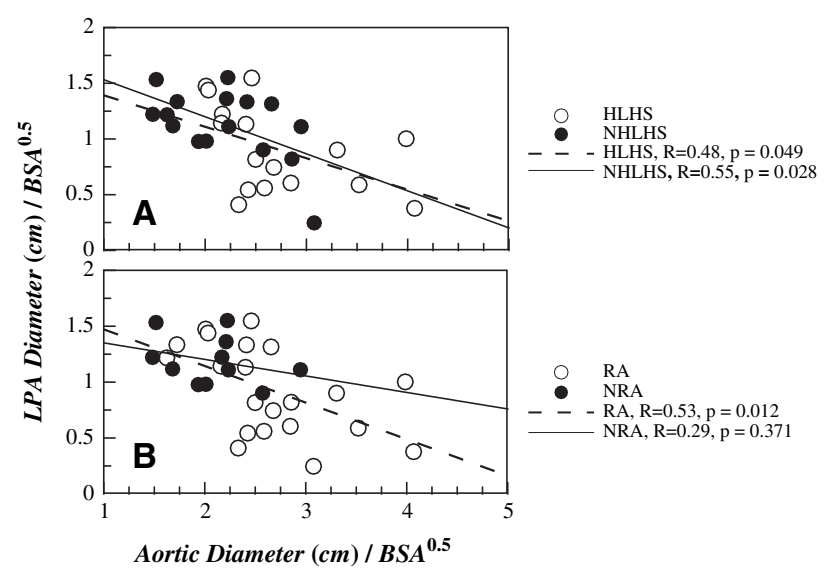

FIGURE 5. Indexed left pulmonary artery ( $L P A)$ diameter plotted against indexed aortic diameter at the junction, with regression analysis shown for the hypoplastic left heart syndrome (HLHS) and non-hypoplastic left heart syndrome $(N H L H S)$ patient groups $(\mathrm{A})$ and patients with reconstructed arteries $(R A)$ and nonreconstructed arteries $(N R A)(\mathrm{B})$, respectively.

when compared with that for the NHLHS group $(P<.05)$. Analysis also showed that the aortic diameter was larger in the RA group compared with that seen in the NRA group $(P<.01)$. This might be explained because the dataset also showed it is more probable that a patient with HLHS has an RA, with a probability of $93.75 \%$. In stark comparison, the probability that a patient with NHLHS has an RA is only $37.50 \%$. Therefore the statistical significance for the larger aorta in Figure 4, $A$, is due to the confounding variable of whether the aorta was reconstructed.

However, this still leaves the question of whether an enlarged aorta constricts the LPA. To answer this question, we performed regression analysis, as depicted in Figure 5. Figure 5, A, shows that in both the HLHS and NHLHS groups, increased aortic size resulted in decreased LPA size. However, as shown in Figure 5, B, increased aortic size resulted in a statistically significant reduction in LPA size only for the RA patient group $(P=.012)$. Moreover, for the NRA group, there was no statistically significant correlation, with a high $P$ value of .37. This implies that narrowing of the LPA is only related to RAs and the underlying defect (HLHS or NHLHS) does not play a role.

Although this study shows how important stage 1 reconstruction can be in the development of LPA in patients undergoing the Fontan operation, it also brings to attention the following question: What is a good aortic reconstruction size? Although this study does not provide a direct answer, we do want to mention that enlarged aortas, such as those discussed above, with a cross-sectional area more than twice the size of a typical adult transverse aorta will contribute to increased hemodynamic energy loss. Given that, we recommend that the RA be no larger than a healthy adult aorta. An additional parameter that can mitigate the constriction of the LPA by the aorta is the length of the aortic arch. A longer length would force the arch to shift in the superior direction, thus relatively moving away from the LPA.

\section{CONCLUSION AND SIGNIFICANCE}

It has been shown that aortic reconstruction that takes place in stage 1 of the 3 palliative surgical procedures that result in Fontan physiology plays a crucial role in LPA growth. Specifically, an enlarged aorta might contribute to diminished LPA size, possibly because of imposed spatial constraints. RAs with more than twice the flow area of an adult aorta were noted in these young patients, and this demonstrates that there needs to be an upper limit placed on the allowable size of an RA in pediatric patients. An enlarged aorta might not only cause LPA stenosis but also in itself create significant energy losses because of the expansion and contraction of the flow, thus further increasing the energetic load on the single ventricle.

\section{References}

1. Disessa TG, Yeatman LA, Williams RG, Lois JF, Friedman WF, Laks H. Thrombosis complicating balloon angioplasty of left pulmonary artery stenosis after Fontan procedure-successful treatment with intravenous streptokinase. Am J Cardiol. 1985;55:610-1.

2. Lock JE, Castanedazuniga WR, Fuhrman BP, Bass JL. Balloon dilation angioplasty of hypoplastic and stenotic pulmonary arteries. Circulation. 1983;67:962-7.

3. Pekkan K, Kitajima HD, de Zelicourt D, Forbess JM, Parks WJ, Fogel MA, et al. Total cavopulmonary connection flow with functional left pulmonary artery stenosis-angioplasty and fenestration in vitro. Circulation. 2005;112:3264-71.

4. Senzaki H, Masutani S, Kobayashi J, Kobayashi T, Sasaki N, Asano H, et al. Ventricular afterload and ventricular work in Fontan circulation-comparison with normal two-ventricle circulation and single-ventricle circulation with BlalockTaussig shunts. Circulation. 2002;105:2885-92.

5. Sundareswaran KS, Pekkan K, Dasi LP, Whitehead K, Sharma S, Kanter KR, et al. The total cavopulmonary connection resistance: a significant impact on single ventricle hemodynamics at rest and exercise. Am J Physiol Heart Circ Physiol. 2008;295:H2427-35.

6. Pekkan K, Dasi L, de Zélicourt D, Sundareswaran K, Fogel M, Kanter K, et al. Hemodynamic performance of stage-2 univentricular reconstruction: Glenn vs. hemi-Fontan templates. Ann Biomed Eng. 2009;37:50-63.

7. Whitehead KK, Pekkan K, Kitajima HD, Paridon SM, Yoganathan AP, Fogel MA. Nonlinear power loss during exercise in single-ventricle patients after the Fontan-insights from computational fluid dynamics. Circulation. 2007;116:I165-71.

8. Dasi LP, KrishnankuttyRema R, Kitajima HD, Pekkan K, Sundareswaran K, Fogel M, et al. Fontan hemodynamics: importance of pulmonary artery diameter. J Thorac Cardiovasc Surg. 2009;137:560-4

9. Senzaki H, Isoda T, Ishizawa A, Hishi T. Reconsideration of criteria for the Fontan operation-influence of pulmonary-artery size on postoperative hemodynamics of the Fontan operation. Circulation. 1994;89:266-71.

10. KrishnankuttyRema R, Dasi LP, Pekkan K, Sundareswaran K, Fogel M, Sharma S, et al. Quantitative analysis of extracardiac versus intraatrial Fontan anatomic geometries. Ann Thorac Surg. 2008;85:810-7.

11. Sundareswaran KS, Kanter KR, Kitajima HD, Krishnankutty R, Sabatier JF, Parks WJ, et al. Impaired power output and cardiac index with hypoplastic left heart syndrome: a magnetic resonance imaging study. Ann Thorac Surg. 2006; 82:1267-77.

12. Frakes DH, Conrad CP, Healy TM, Monaco JW, Fogel M, Sharma S, et al. Application of an adaptive control grid interpolation technique to morphological vascular reconstruction. IEEE Trans Biomed Eng. 2003;50:197-206.

13. Frakes DH, Smith MJT, Parks J, Sharma S, Fogel M, Yoganathan AP. New techniques for the reconstruction of complex vascular anatomies from MRI images. J Cardiovasc Magn Res. 2005;7:425-32.

14. Sluysmans T, Colan SD. Theoretical and empirical derivation of cardiovascular allometric relationships in children. J Appl Physiol. 2005;99:445-57. 